\title{
A multidomain approach of the Fourier pseudospectral method using discontinuous grid for elastic wave modeling
}

\author{
Yanbin Wang* and Hiroshi Takenaka \\ Department of Earth and Planetary Sciences, Kyushu University, Hakozaki 6-10-1, Fukuoka 812-8581, Japan
}

(Received June 19, 2000; Revised January 22, 2001; Accepted January 25, 2001)

\begin{abstract}
Pseudospectral method is an efficient and high-accuracy numerical modeling technique that has been applied to the simulation of seismic wavefields in realistic models with complex structures such as sedimentary basin. However, if the sedimentary layers are included in the model, high velocity contrasts arise because of the very low $S$-wave velocities of the sediments as compared with the bedrock. The grid spacing then has to be very small, which leads to spatial oversampling in regions with higher velocities. As a result, we also have to use very small time interval for time marching, so that the computation requires much CPU time. A treatment of this problem is use of a multidomain approach that can employ different grid spacings in different regions, i.e. "discontinuous grid". In this study, we propose a scheme for the Fourier pseudospectral method using discontinuous grid, which uses an interpolation by the FFT. This scheme can reduce the computation time and the required computer memory as compared with the conventional one. The accuracy and efficiency of this approach are demonstrated in the present paper through examples of 2-D elastic wave modeling.
\end{abstract}

\section{Introduction}

Pseudospectral method (PSM) and the finite difference method (FDM) are frequently used in the simulations of seismic wave propagation in realistic structures because of their high-accuracy and efficiency. Recently, the FDM has been applied to the simulations of seismic wavefields in 3-D structure models excited by real earthquakes (e.g., Olsen et al., 1995; Olsen and Archuleta, 1996; Graves, 1996; Sato et al., 1998). However, due to the large requirements of computer memory and the computation time caused by a great number of the spatial grid points, the 3-D simulations could only be performed for rather long period seismic waves in model with large grid spacing. Therefore, very low velocity sedimentary layers just below the free surface could not be realistically incorporated into the model. In order to include very low velocity surface layers into the model and make the computation to be performed efficiently, several approaches based on the use of an irregular grid (Moczo, 1989; Moczo and Bard, 1993; Pitarka, 1999) or a discontinuous grid (Jastram and Behle, 1992; Jastram and Tessmer, 1994; Falk et al., 1996; Moczo et al., 1996, 1997; Aoi and Fujiwara, 1999) were proposed for the FDM that can effectively reduce the unnecessary grid points in the high-velocity region in the model and thus reduce the computational requirements in the computer memory and computation time.

To achieve the same accuracy, the PSM (e.g., Kosloff and Baysal, 1982; Kosloff et al., 1984) needs less grid points and

*Now at Département de Sismologie, Institut de Physique du Globe de Paris, 4 Place Jussieu, 75252 Paris Cédex 05, France.

Copy right $(\mathrm{C})$ The Society of Geomagnetism and Earth, Planetary and Space Sciences (SGEPSS); The Seismological Society of Japan; The Volcanological Society of Japan; The Geodetic Society of Japan; The Japanese Society for Planetary Sciences. therefore uses less computer memory and computation time compared with the FDM for models with smoothly varying heterogeneity (e.g., Fornberg, 1987; Daudt et al., 1989). The advantages of the PSM make it practically effective to apply the method to the simulation of seismic wavefield in realistic model that has complex structure. Several studies presented the applications of this method to 3-D models (e.g., Furumura et al., 1996, 1998a; Furumura and Koketsu, 1998; Hung and Forsyth, 1998). The advantages of the PSM arise from the spatial sampling that less grid points are needed for the shortest wavelength than the FDM, and the grid spacing used in the PSM is then much larger as compared with that in the FDM. However, there are also drawbacks in the PSM, that is, the large grid spacing in a conventional uniform grids in the PSM fails to represent the detailed variation of the very low velocity layers near the surface and the interfaces. Compared with the FDM, it is more difficult to include the very low velocity surface layers into the model in the conventional PSM using uniform grids.

In this paper, we present a multidomain technique that discretizes the entire region of the model with discontinuous grid. By using the multidomain, the whole model is divided into several subdomains with different grid spacings. The very low velocity region is discretized with a smaller grid spacing while the high-velocity region with a larger grid spacing. With this scheme, we keep the advantages of the pseudospectral method in accuracy and efficiency, and the drawbacks caused by the coarse grid spacing in the conventional uniform grids are removed, so that the very low velocity region in the model can be represented more realistically.

In the next section we describe our multidomain scheme for the PSM. We then demonstrate the implementation and accuracy of the scheme using a 2-D model in Section 3, and 
(a)

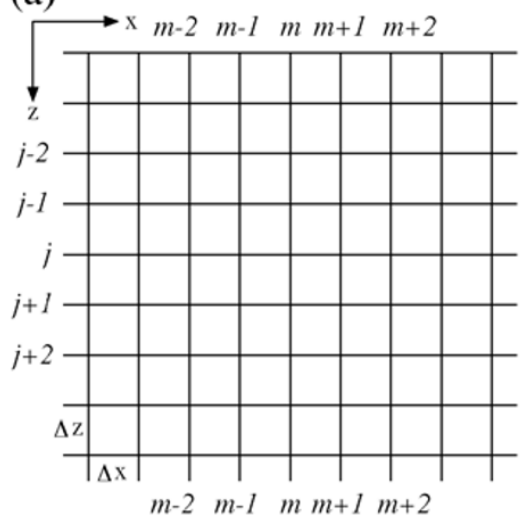

(b)

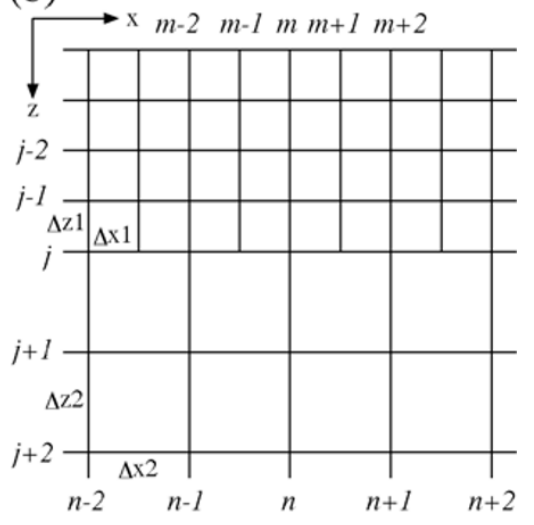

Fig. 1. Configuration of the grids in 2-D Cartesian coordinate system $(x, z)$. (a) The uniform grid in which the entire region of the model is discretized with the same grid spacing in each coordinate. $\Delta x$ and $\Delta z$ are the grid spacing in $x$ and $z$ directions, respectively. (b) The discontinuous grid used in the multidomain scheme in which the grid spacing varies with the velocity from region to region. The region with lower wave velocity has smaller grid spacing than that with higher wave velocity. $\Delta x_{1}$ and $\Delta z_{1}$ are the grid spacings in the low-velocity region in the $x$ and $z$ directions, $\Delta x_{2}$ and $\Delta z_{2}$ are the grid spacings in the high-velocity region in the $x$ and $z$ directions.

show two examples of 2-D elastic modeling to illustrate the efficiency of the scheme. In this paper we will show only 2-D calculations due to limitation of our available computational resources. But the implementation of our scheme to 3-D modeling is straightforward and has no additional technical problems. The last section of this paper gives conclusions.

\section{Multidomain Scheme}

For simplicity, we consider a model defined in a 2-D Cartesian coordinate system $(x, z)$ as displayed in Fig. 1 to describe our mutidomain scheme. In the conventional PSM algorithm, the entire region of the model is discretized in a uniform grid as shown in Fig. 1(a). The numbers of grid points in both the $x$ and $z$ directions are constant, the entire region is discretized with constant grid spacings in both the $x$ and $z$ directions, and the grids are continuous in both axes throughout the model. The grid size is determined by the minimum wavelength in the region with the lowest wave velocity. If such a uniform grid is used to represent the velocity variation in the very low velocity region, it then causes spatial oversampling in the higher velocity region. Application of the mapping technique in both $x$ and $z$ directions as suggested by Fornberg (1988), Jastram and Tessmer (1994) and Furumura et al. (1998b) can re-locate the grids by following the interface and reduce the grid spacing at the very low velocity layers to improve the accuracy of the results. But the total number of grid points remains the same and the spatial oversampling in high-velocity region is not removed.

To overcome the spatial oversampling, we use the discontinuous grid as shown in Fig. 1(b) to discretize the model. The entire region is divided into several subdomains which have different grid spacings in the $x$ and $z$ directions and the numbers of grid points vary between the subdomains. The low-velocity region is discretized by $\Delta x_{1}$ and $\Delta z_{1}$, where $\Delta x_{1}$ and $\Delta z_{1}$ may vary with $x$ and $z$. The $\Delta x_{1}$ and $\Delta z_{1}$ are determined by the shortest wavelength in this region. In the high-velocity region, the model is discretized with larger grid spacings $\Delta x_{2}$ and $\Delta z_{2}$, which are determined by the shortest wavelength in this region.
On the conventional uniform grid shown in Fig. 1(a), the numbers of grid points in both the $x$ and $z$ directions are constant. The grid points are continuous throughout the whole model. Calculations of the spatial derivatives of field variables in the wave equations are performed along both $x$ and $z$ axes for all the grid points. The spatial derivatives with respect to $x$ are evaluated for all the grid points $m$, and to $z$ for all the grid points $j$ using the FFT. On the discontinuous grid shown in Fig. 1(b), the spatial derivatives with respect to $x$ are evaluated in the first subdomain for grid points $m$, in the second subdomain for grid points $n$. The spatial derivatives with respect to $z$ can be calculated for all grid points $j$ at $m-2, m, m+2$ in the first subdomain and at the corresponding locations $n-1, n, n+1$ in the second subdomain. However, the grid points in the $z$ direction at $m-1, m+1$ are defined only in the first subdomain. In order to calculate the spatial derivatives with respect to $z$ on grid points $j$ at $m-1, m+1$, it is necessary to evaluate the values of the field variables half-way between grid points $n-1$ and $n, n$ and $n+1$ in the second subdomain. The spatial derivatives with respect to $z$ for grid points $j$ at $m-1, m+1$ in the first subdomain can therefore be calculated for the field variables on these grid points and the values at the corresponding locations $n-1 / 2, n+1 / 2$ in the second subdomain. We will calculate the values of field variables at $n \pm 1 / 2$ in the coarser grid using interpolation.

Let the number of grid points and grid spacing be $N_{1}$ and $\Delta x_{1}$ in the finer grid, and $N_{2}$ and $\Delta x_{2}$ in the coarser grid. The Fourier transform of a field variable $g\left(n \Delta x_{2}\right)$ $\left(n=0,1, \ldots, N_{2}-1\right)$ in the subdomain of high velocity is

$$
G\left(l_{2} \Delta k_{2}\right)=\Delta x_{2} \sum_{n=0}^{N_{2}-1} g\left(n \Delta x_{2}\right) e^{-\mathrm{i} 2 \pi l_{2} n / N_{2}} .
$$

Its Nyquist wavenumber is

$$
N_{\mathrm{q} 2}=\frac{\pi}{\Delta x_{2}} .
$$

Interpolate the $g\left(n \Delta x_{2}\right)$ into $g\left(m \Delta x_{1}\right)(m=0,1, \ldots$, 
$\left.N_{1}-1\right)$. Then the Fourier transform of $g\left(m \Delta x_{1}\right)$ is

$$
G\left(l_{1} \Delta k_{1}\right)=\Delta x_{1} \sum_{m=0}^{N_{1}-1} g\left(m \Delta x_{1}\right) e^{-\mathrm{i} 2 \pi l_{1} m / N_{1}},
$$

with Nyquist wavenumber defined as

$$
N_{\mathrm{q} 1}=\frac{\pi}{\Delta x_{1}} .
$$

Since $\Delta x_{2}$ is larger than $\Delta x_{1}, N_{\mathrm{q} 1}$ is greater than $N_{\mathrm{q} 2}$. By setting the wavenumber components between $N_{\mathrm{q} 2}$ and $N_{\mathrm{q} 1}$ equal to zeros, we get the Fourier transform $G\left(l_{1} \Delta k_{1}\right)\left(l_{1}=\right.$ $\left.0,1, \ldots, N_{1}-1\right)$ for the interpolated function $g\left(m \Delta x_{1}\right)$ from the Fourier transform $G\left(l_{2} \Delta k_{2}\right)\left(l_{2}=0,1, \ldots, N_{2}-1\right)$ of the original $g\left(n \Delta x_{2}\right)$. The interpolated function $g\left(m \Delta x_{1}\right)$ is then obtained from the inverse Fourier transform of $G\left(l_{1} \Delta k_{1}\right)$ as

$$
g\left(m \Delta x_{1}\right)=\frac{\Delta k_{1}}{2 \pi} \sum_{l_{1}=0}^{N_{1}-1} G\left(l_{1} \Delta k_{1}\right) e^{\mathrm{i} 2 \pi l_{1} m / N_{1}} .
$$

After interpolation, the values of field variables on grid points $n-1, n, n+1$ in the coarser grid remain unchanged and the values between the grid points at $n \pm 1 / 2$ are obtained, which are used for calculating the spatial derivatives with respect to $z$ on grid points $j$ at $m-1, m+1$ in the $z$ direction. The number of grid points in the finer grid in the $x$ direction is two or four times that in the coarser grid, and the interpolation in the coarser grid can be performed in the wavenumber domain using the FFT.

The values of field variables between grid points can also be calculated using a shift of the field variables in the space domain by the FFT. If $N_{1}$ is equal to $2 N_{2}$, the values at $\left(n \pm \frac{1}{2}\right) \Delta x_{2}$ in $x$ direction in the coarser grid in Fig. 1(b) can be obtained by shifting the values on grid points in $x$ direction by $\Delta x_{2} / 2$. The shift in the space domain corresponds to a multiplication in the wavenumber domain

$$
g\left(x \pm \frac{\Delta x_{2}}{2}\right)=\int_{-\infty}^{\infty} G(k) e^{\mathrm{i} k\left(x \pm \Delta x_{2} / 2\right)} d k,
$$

where $G(k)$ is the Fourier transform of the function $g(x)$ and the interpolation operator is given by

$$
D_{x}(k)=e^{ \pm \mathrm{i} k \Delta x_{2} / 2},
$$

where "+" indicates the shift in positive and "-" in negative $x$ direction.

\section{Implementation and Accuracy Test of the Scheme}

In this section, we demonstrate the implementation and accuracy of the multidomain scheme described in the previous section using a 2-D model calculation. The model used in this calculation is a homogeneous half-space with seismic wave velocity $V_{P}=6.93 \mathrm{~km} / \mathrm{s}, V_{S}=4.0 \mathrm{~km} / \mathrm{s}$ and density $\rho=2.6 \mathrm{~g} / \mathrm{cm}^{3}$ as shown in Fig. 2(a).

We consider the in-plane motion in the $x-z$ plane with the equation of motion

$$
\begin{aligned}
& \rho \frac{\partial^{2} u_{x}}{\partial t^{2}}=\frac{\partial \sigma_{x x}}{\partial x}+\frac{\partial \sigma_{z x}}{\partial z}+f_{x}, \\
& \rho \frac{\partial^{2} u_{z}}{\partial t^{2}}=\frac{\partial \sigma_{x z}}{\partial x}+\frac{\partial \sigma_{z z}}{\partial z}+f_{z},
\end{aligned}
$$

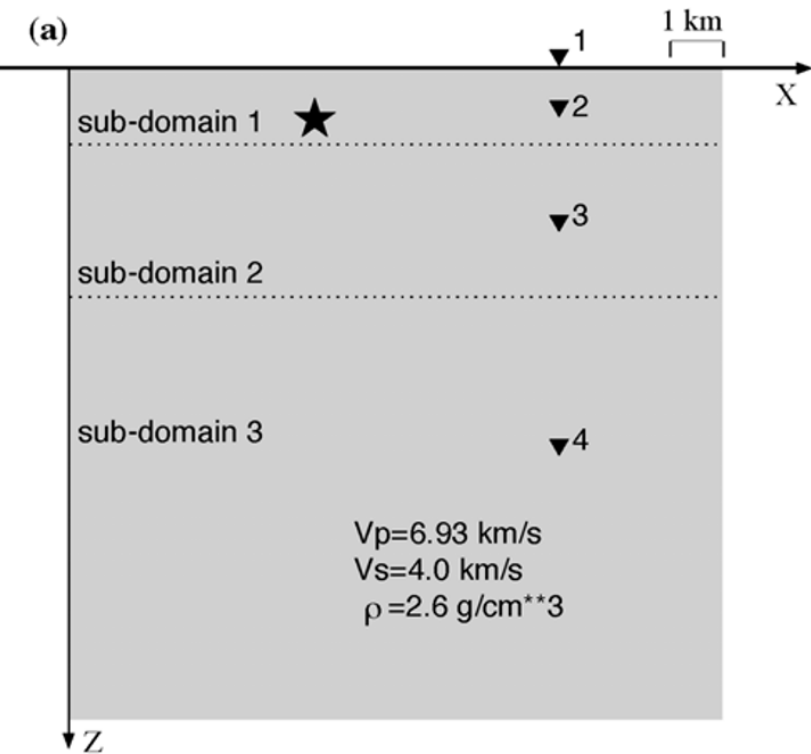

(b)

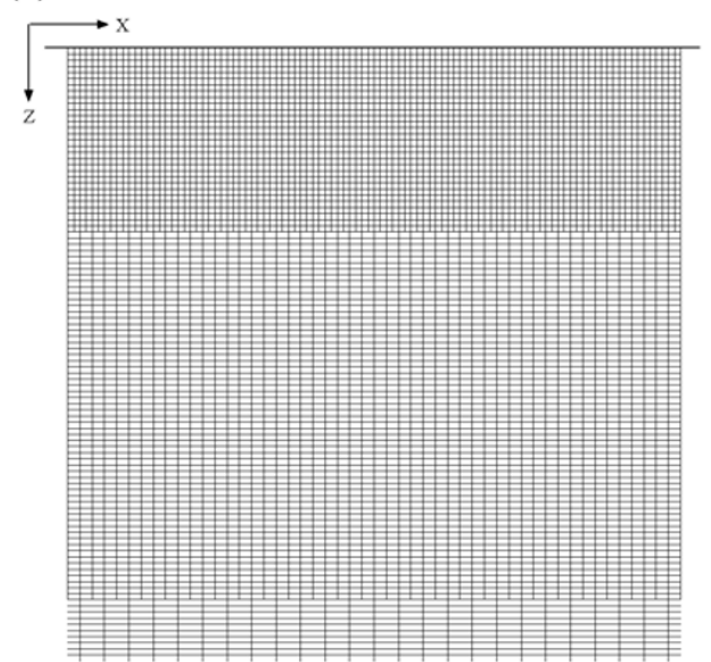

Fig. 2. The homogeneous half-space model used for the calculation to check the accuracy of the multidomain scheme. (a) The whole model is divided into three subdomains. Dotted lines are the boundaries between subdomains. Star and inverted triangles indicate the locations of the seismic source and the observation points, respectively. (b) Part of the grid for the model. The grid spacing $\Delta x$ is the same in each subdomain but increases twice between subdomains. The grid spacing $\Delta z$ is the same for all the model in the vertical direction.

and the following stress-strain relations

$$
\begin{aligned}
& \sigma_{x x}=(\lambda+2 \mu) \frac{\partial u_{x}}{\partial x}+\lambda \frac{\partial u_{z}}{\partial z}, \\
& \sigma_{z z}=(\lambda+2 \mu) \frac{\partial u_{z}}{\partial z}+\lambda \frac{\partial u_{x}}{\partial x}, \\
& \sigma_{x z}=\sigma_{z x}=\mu\left(\frac{\partial u_{x}}{\partial z}+\frac{\partial u_{z}}{\partial x}\right),
\end{aligned}
$$

where $u_{x}$ and $u_{z}$ are displacement components, $\sigma_{x x}, \sigma_{x z}, \sigma_{z x}$ and $\sigma_{z z}$ are stress-tensor components, $f_{x}$ and $f_{z}$ are body force components in the $x$ and $z$ direction, $\rho$ is density, $\lambda$ and $\mu$ are the Lamé parameters.

Using a discontinuous grid, the scheme to solve the above equations in each time step can be summarized as follows: 

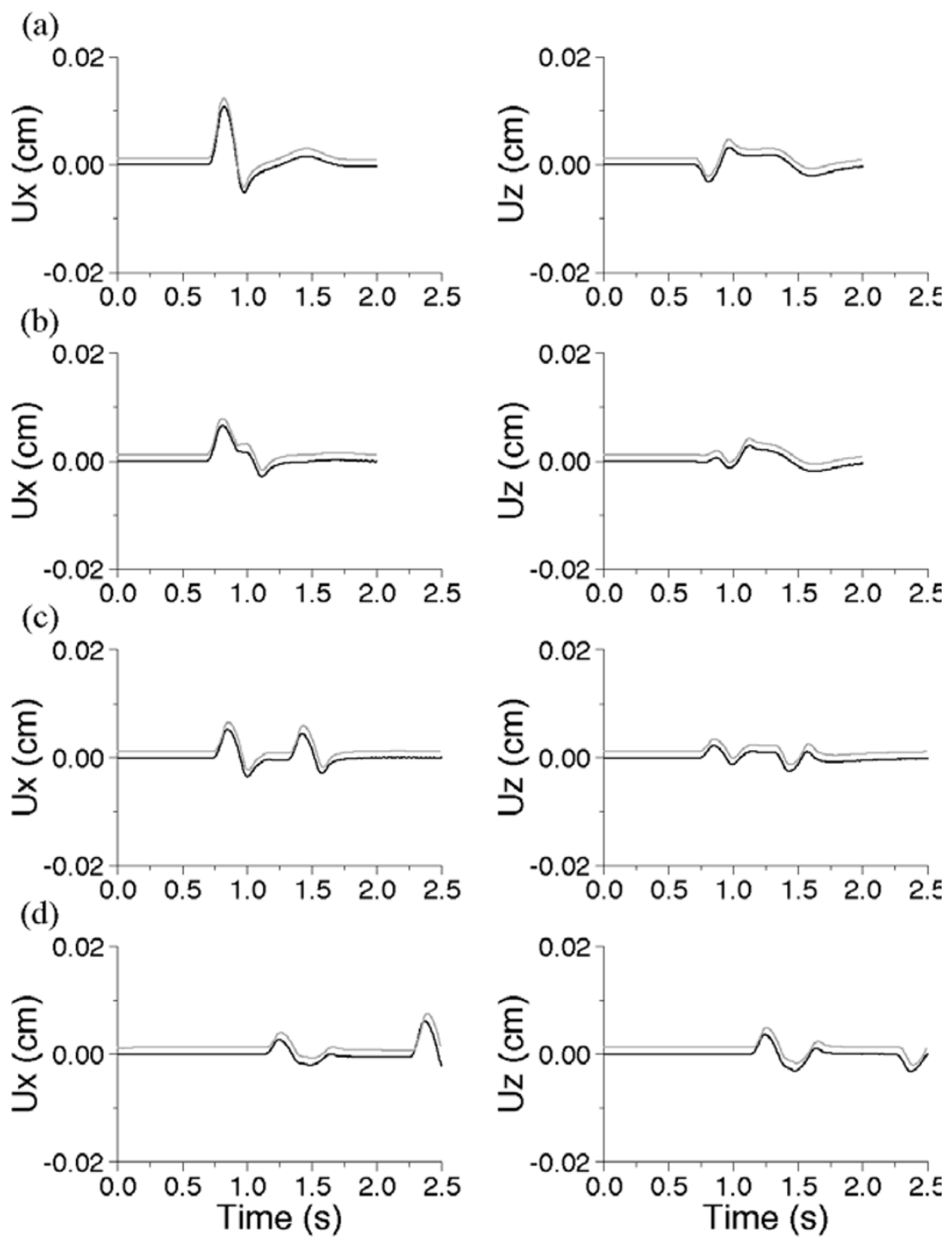

Fig. 3. Comparison of the synthetic seismograms on four observation points as shown in Fig. 2(a) calculated by the multidomain scheme (heavy lines) and those obtained from the discrete wavenumber method (dashed lines).

1. Calculate the spatial derivatives $\partial / \partial x$ for $u_{x}$ and $u_{z}$ at all grid points in each subdomain,

2. Perform interpolation to evaluate the values of field variables $u_{x}$ and $u_{z}$ between grid points in the subdomains with coarser grid,

3. Calculate the spatial derivatives $\partial / \partial z$ for $u_{x}$ and $u_{z}$ at all grid points and the interpolated values in the whole range in the $z$ direction,

4. Calculate the stress components in Eq. (9),

5. Perform steps 1 to 3 for the stress components,

6. Calculate the $\partial^{2} u_{x} / \partial t^{2}$ and $\partial^{2} u_{z} / \partial t^{2}$ in Eq. (8),

7. Perform time integration to obtain the displacement components $u_{x}$ and $u_{z}$,

8. Repeat the above operations in the next time step.
In this calculation, the whole model in Fig. 2(a) is divided into three subdomains in the vertical direction. The grid spacing $\Delta z$ in the vertical direction is $0.05 \mathrm{~km}$ for all subdomains. The grid spacing $\Delta x$ in the horizontal direction in the three subdomains from the top surface to the bottom is 0.05 $\mathrm{km}, 0.1 \mathrm{~km}$ and $0.2 \mathrm{~km}$, respectively. The numbers of grid points in the horizontal and vertical directions are 512 and 30 in the upper-most subdomain, 256 and 60 in the middle, and 128 and 166 in the lowest subdomain. Part of the grid is schematically displayed in Fig. 2(b).

The free surface boundary condition is incorporated into the calculation by using the symmetric differentiation for the displacement components and the anti-symmetric differentiation for the stress components when calculating the spatial derivatives with respect to $z$ (Furumura and Takenaka, 1992). The absorbing boundary condition proposed by Cerjan et al. (1985) is used for all other boundaries by setting the width of the damping zone to 20 grid points and the damping coefficient to 0.015 . Since $\Delta x$ increases twice between sub- 
domains in the $z$ direction, the actual widths of the damping zone in the three subdomains are $1.0 \mathrm{~km}, 2.0 \mathrm{~km}$ and 4.0 $\mathrm{km}$, respectively, for the boundaries of the model in the $x$ direction.

The seismic source used in the calculation is an explosion point source located at $1.0 \mathrm{~km}$ depth with moment-tensor components $M_{x x}=1.0 \times 10^{12} \mathrm{Nm}$ and $M_{z z}=1.0 \times 10^{12}$ $\mathrm{Nm}$. The body-force components corresponding to the explosion source are distributed in both the $x$ and $z$ directions with a pseudo-delta function (Herrmann, 1979). The sourcetime function is also approximated by the same type pseudodelta function with a width of $0.3 \mathrm{~s}$. The time interval used in the calculation is $0.001 \mathrm{~s}$ that is determined from the minimum grid spacing $0.05 \mathrm{~km}$ and the maximum seismic wave velocity in the model. The calculation is carried out for 3000 time steps to get the synthetic seismograms with duration of $3 \mathrm{~s}$.

Figure 3 shows the comparison of the synthetic seismograms with those calculated by the discrete wavenumber method (Bouchon and Aki, 1977). At all the four observation points, very good agreements in both the amplitudes and the arrival times can be seen. The comparison suggests that the multidomain scheme using discontinuous grid can give sufficiently accurate results.

In this calculation model the widths of the side absorbing boundaries increase twice between adjoining subdomains as mentioned above. If the same width of damping zone were used in the three subdomains, for example, $1.0 \mathrm{~km}$ in all $z$, the numbers of grid points in the damping zone would be 10 and 5 in the second and third subdomain that could not effectively eliminate the wraparound in the $x$ direction in these two subdomains. In our calculation model with the side absorbing boundaries that are not uniform at the interfaces between subdomains, the wraparound in the $x$ direction in each subdomain can be effectively removed by the damping zones. In the $z$ direction, the wraparound can also be effectively removed by the damping zone along the whole bottom boundary since the spatial derivatives of the field variables with respect to $z$ are calculated for all subdomains along the whole range in $z$. The differential width of the damping zones causes differential damping of the values of field variables in the $z$ direction at both side boundaries, but does not bring problems of wraparound. This can be seen in the results of examples in the following sections. But the wide damping zones in the $x$ direction reduces the effective region of the wavefield to be simulated in the deeper area with higher velocity. If we are interested in the wavefield of the high-velocity deeper area, a wide enough effective space has to be used in the model in the $x$ direction, which will increase the number of grid points in the $x$ direction and degrade the efficiency of the multidomain scheme.

\section{Efficiency of the Scheme}

In this section, we illustrate the efficiency of the multidomain scheme by the following two examples of 2-D elastic modeling.

\subsection{Example 1}

We use a 1-D heterogeneous model composed of two layers over a half-space as shown in Fig. 4. The top layer is a sedimentary layer that has very low seismic wave veloc-

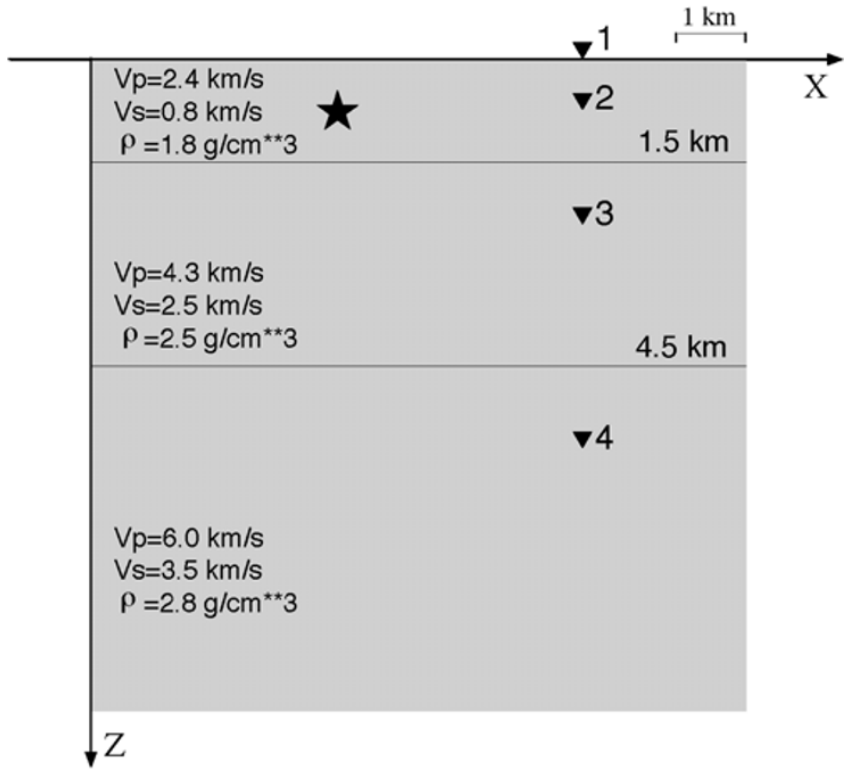

Fig. 4. The vertically heterogeneous model used for the calculation to show the efficiency of the multidomain scheme. The model is composed of two layers over a half-space. Star and inverted triangles indicate the locations of the seismic source and the observation points.

ities $V_{P}=2.4 \mathrm{~km} / \mathrm{s}, V_{S}=0.8 \mathrm{~km} / \mathrm{s}$ and density $\rho=1.8$ $\mathrm{g} / \mathrm{cm}^{3}$. The seismic wave velocities and density in the middle layer are $V_{P}=4.3 \mathrm{~km} / \mathrm{s}, V_{S}=2.5 \mathrm{~km} / \mathrm{s}$ and $\rho=2.5$ $\mathrm{g} / \mathrm{cm}^{3}$, and $V_{P}=6.0 \mathrm{~km} / \mathrm{s}, V_{S}=3.5 \mathrm{~km} / \mathrm{s}$ and $\rho=2.8$ $\mathrm{g} / \mathrm{cm}^{3}$ in the half-space. The thickness of the top and middle layers are $1.5 \mathrm{~km}$ and $3.0 \mathrm{~km}$, respectively. The model is divided into three subdomains according to the velocity distribution. The first subdomain is the low-velocity top layer. Numbers of grid points in the $x$ and $z$ directions are 512 and 30 , respectively, with grid spacings $\Delta x_{1}=\Delta z_{1}=0.05 \mathrm{~km}$. The second subdomain is the middle layer. Numbers of grid points in the $x$ and $z$ directions are 256 and 30, grid spacings are $\Delta x_{2}=\Delta z_{2}=0.1 \mathrm{~km}$. The third subdomain is the halfspace. Numbers of grid points in the $x$ and $z$ directions are 128 and 196, grid spacings are $\Delta x_{3}=\Delta z_{3}=0.2 \mathrm{~km}$.

To check the efficiency of the multidomain scheme, we perform another simulation for the same model by employing a uniform grid. In the uniform grid, only a single domain is used for whole region of the model. The numbers of grid points are then 512 and 256 in the $x$ and $z$ directions, respectively. The grid spacings are the same as those used in the first subdomain in the discontinuous grid with $\Delta x=$ $\Delta z=0.05 \mathrm{~km}$. Also the same seismic source, free surface and absorbing boundary conditions, spatial distribution of the body force components and the source time function are used.

In the uniform grid, the time interval used in the calculation is determined by the following stability condition

$$
\Delta t<\alpha \frac{\Delta^{\min }}{V^{\max }},
$$

where $\Delta^{\text {min }}$ is the minimum grid spacing in the whole grid, $V^{\max }$ is the maximum seismic wave velocity in the whole model and $\alpha$ is a coefficient that defines the accuracy in the 

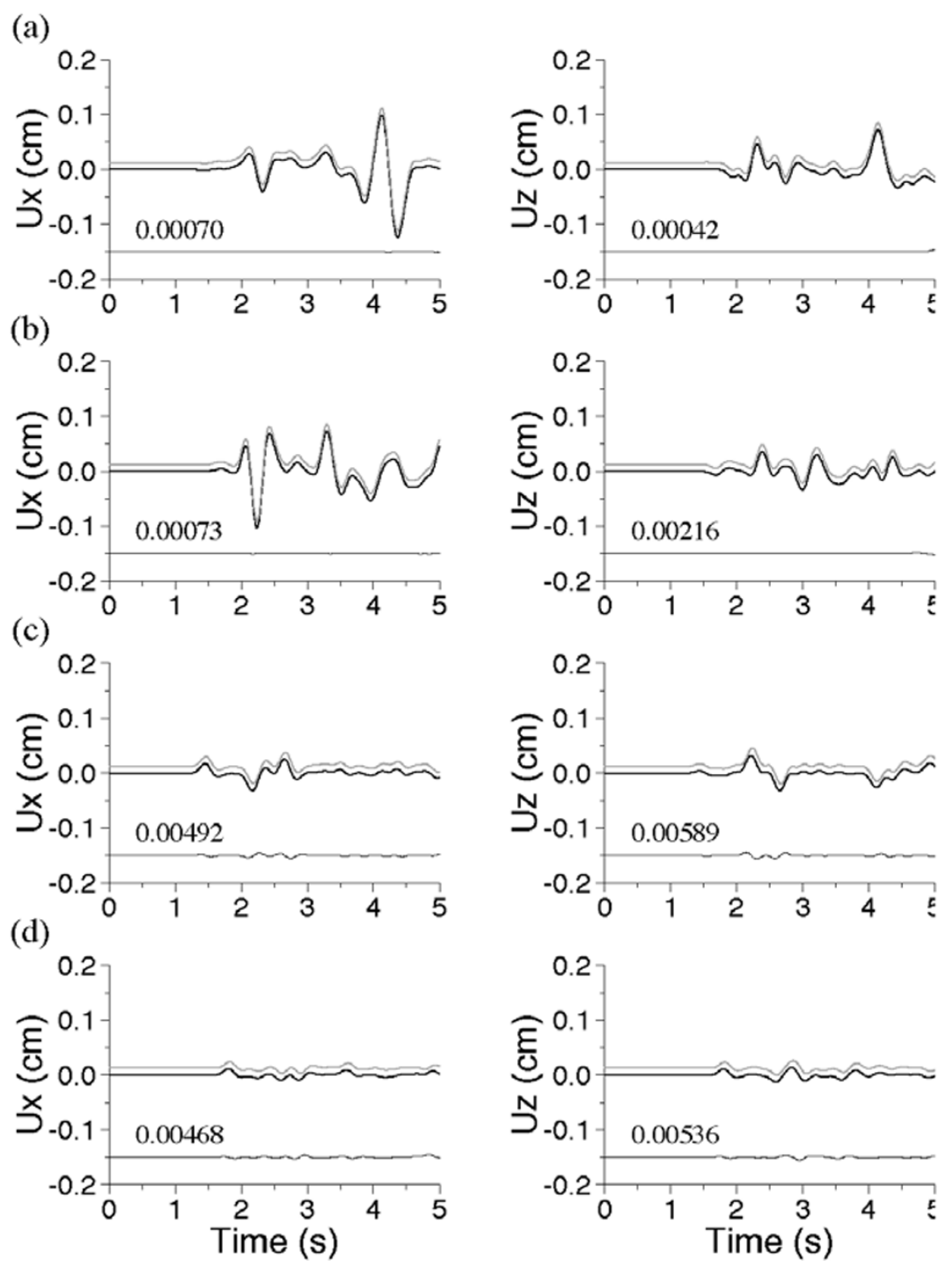

Fig. 5. Comparison of the synthetic seismograms at four observation points as shown in Fig. 4 calculated using the discontinuous grid (heavy lines) and those obtained using the uniform grid (dashed lines). The thin lines in the lower part are the differential seismograms between the two results marked with the maximum value.

time integration calculation. In the discontinuous grid, the time interval in each subdomain is determined by the $\Delta^{\text {min }}$ and $V^{\max }$ in the subdomain using the above stability criterion. We choose the minimum one in all subdomains, which is larger than the time interval used in the uniform grid. In this calculation, for $\alpha=0.26$ ( $1 \%$ tolerance error level, see Daudt et al. (1989)), the $\Delta t$ calculated using (10) in the three subdomains are less than $0.005 \mathrm{~s}, 0.006 \mathrm{~s}$ and $0.008 \mathrm{~s}$, respectively. We use $\Delta t=0.004 \mathrm{~s}$ in the calculation for the discontinuous grid. The time interval for the uniform grid determined from the minimum grid spacing $(0.05 \mathrm{~km})$, the maximum velocity $(6.0 \mathrm{~km} / \mathrm{s})$ and $\alpha=0.26$ is $0.0022 \mathrm{~s}$.

In the discontinuous grid, the number of grid points in the $x$ direction is reduced by half and $3 / 4$ in the second and third subdomain, respectively, compared to the uniform grid.

The synthetic seismograms calculated for both the uniform and discontinuous grids are displayed in Fig. 5 for comparison. The amplitudes and arrival times of all phases agree very well. This suggests that the multidomain scheme can give the same synthetic results as the single domain scheme,
Table 1. Comparison of the computational requirements for uniform and discontinuous grids.

\begin{tabular}{ccc}
\hline & Discontinuous grid & Uniform grid \\
\hline$\Delta t$ & $0.004 \mathrm{~s}$ & $0.002 \mathrm{~s}$ \\
\hline Seismogram duration & $5.0 \mathrm{~s}$ & $5.0 \mathrm{~s}$ \\
\hline Time steps & 1250 & 2500 \\
\hline CPU time & $37.65 \mathrm{~min}$ & $117.63 \mathrm{~min}$ \\
\hline Memory & $19.71 \mathrm{Mbs}$ & $23.96 \mathrm{Mbs}$ \\
\hline
\end{tabular}

but with less computational requirements. The time interval, time steps, CPU time and computer memory used by both grids are listed for comparison in Table 1. The calculations are performed in single precision on a SUN Ultra 1 workstation with $143 \mathrm{MHz}$ clock speed. The discontinuous grid requires about $32 \%$ of the CPU time and about $82 \%$ of the computer memory compared to the uniform grid. The effi- 

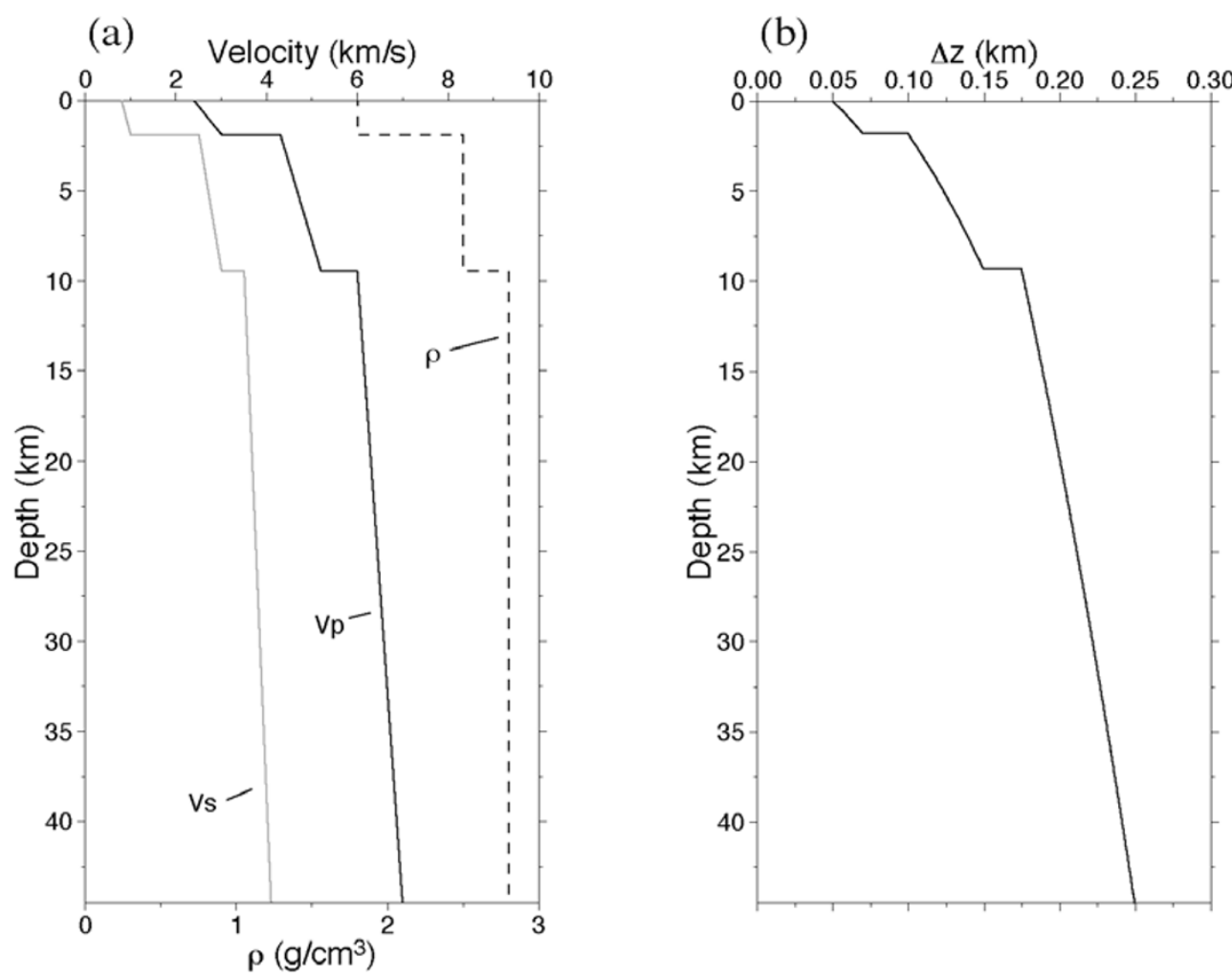

Fig. 6. (a) The velocity and density distribution with depth for the model used in the third example. Both $V_{P}$ and $V_{S}$ increase linearly with depth. The whole model is divided into three subdomains with boundaries located at the velocity discontinuities. (b) The distribution of vertical grid spacing $\Delta z$ with depth. It increases linearly within each subdomain and is discontinuous between the subdomains. The linear increase and discontinuities of $\Delta z$ corresponds to the linear increase and discontinuities of seismic wave velocity in depth.
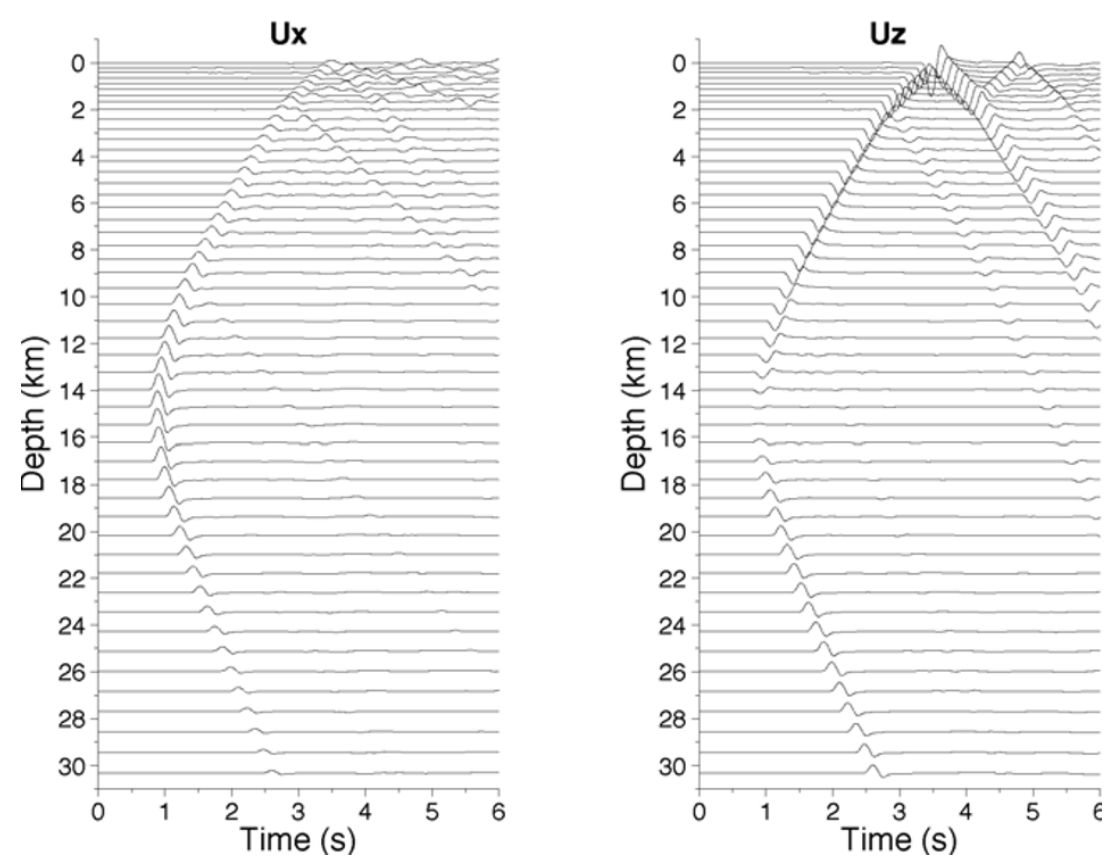

Fig. 7. The synthetic seismograms along a vertical profile at a horizontal offset of $4.8 \mathrm{~km}$ from the source for the realistic model shown in Fig. $6(\mathrm{a})$ computed using discontinuous grid in the $x$ direction and varying grid spacing in the $z$ direction. The explosion source is located at a depth of $15.0 \mathrm{~km}$. 


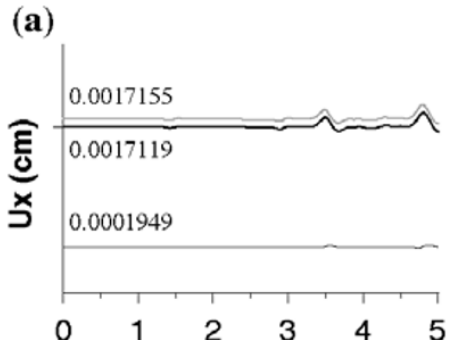

(b)

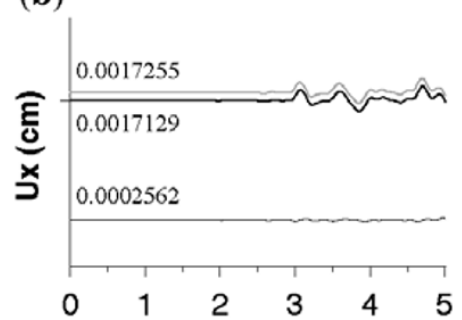

(c)

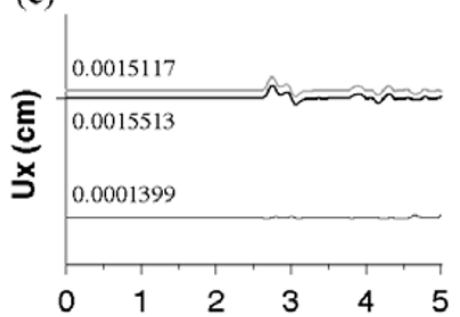

(d)

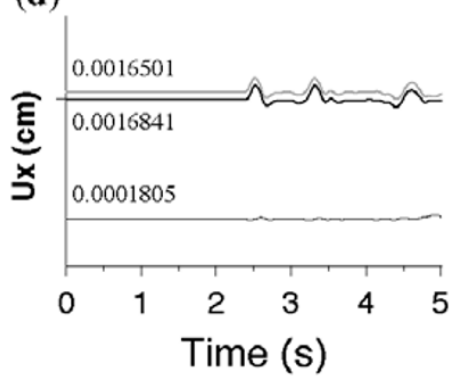

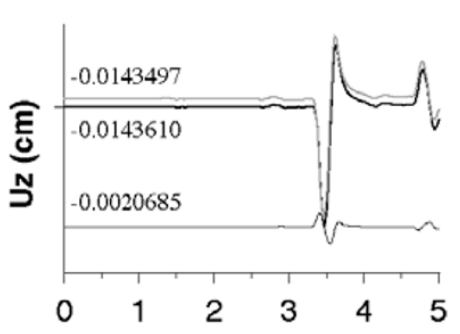
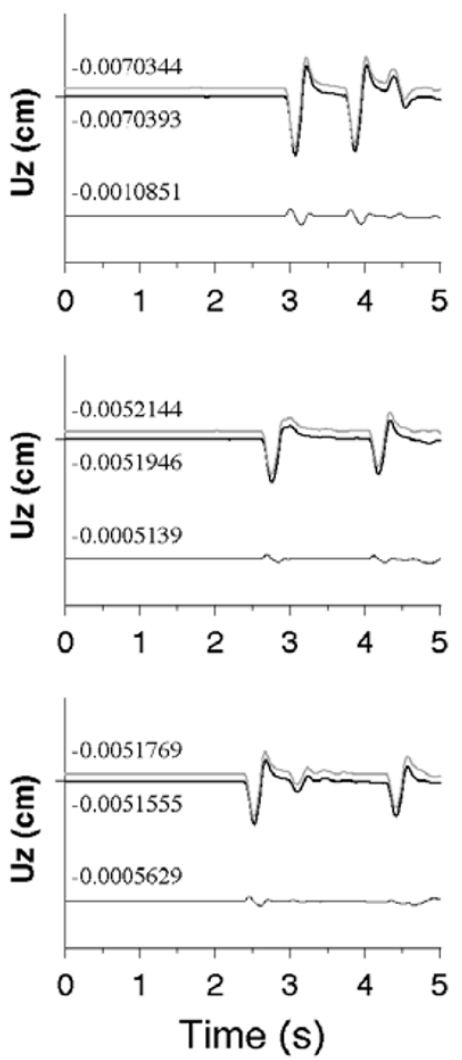

Fig. 8. Comparison of the synthetic seismograms at four observation points at (a) the surface, (b) $1.0 \mathrm{~km}$, (c) $2.0 \mathrm{~km}$, and (d) $3.0 \mathrm{~km}$ depth along the profile shown in Fig. 7 calculated using the discontinuous grid (heavy lines) and those obtained using the uniform grid (dashed lines). The maximum amplitude is written along the traces. The thin lines in the lower part are the differential seismograms between the two results marked with the maximum value.

ciency of the multidomain scheme should be more prominent for large scale or 3-D model.

\subsection{Example 2}

In the model used in Section 3, the grid spacing in the $x$ direction increases in depth in different subdomains, but the grid spacing in the $z$ direction is constant. This makes the shape of the grid cell in the second and third subdomain to be rectangular as shown in Fig. 2(b). The difference between the lengths of the grid cell in the $x$ and $z$ directions then becomes larger with increasing depth. The vertical and horizontal dimensions of a grid cell should be as close as possible throughout the whole model in order to ensure similar spatial sampling of the physical model in the $x$ and $z$ directions. In the example shown in Subsection 4.1, the grid spacings in both the $x$ and $z$ directions are the same in each subdomain that ensures the same spatial sampling in both directions. We here consider a more realistic structure model and construct a more natural discontinuous grid by using the multidomain scheme in the $x$ direction and a mapping technique in the $z$ direction.

The vertically heterogeneous model is composed of two layers over a half-space. Within each layer and the halfspace, the seismic wave velocities and density increase linearly with depth as shown in Fig. 6(a). Such a model usually is more realistic than the homogeneous layer model. The whole model is divided into three subdomains with boundaries located at the discontinuities in the medium. In the first subdomain located just below the surface, the numbers of grid points in the $x$ and $z$ directions are 512 and 30, the grid spacing $\Delta x$ is $0.05 \mathrm{~km}$ and $\Delta z$ varies from $0.05 \mathrm{~km}$ at the top to $0.07 \mathrm{~km}$ at the bottom. In the second subdomain located in the middle of the model, the numbers of the grid points are 256 and 60 , the grid spacing $\Delta x$ is $0.1 \mathrm{~km}$ and $\Delta z$ varies from $0.1 \mathrm{~km}$ at the top to $0.15 \mathrm{~km}$ at the bottom. In the third subdomain located in the half-space, the numbers of the grid points in the $x$ and $z$ directions are 128 and 166, the grid spacing $\Delta x$ is $0.2 \mathrm{~km}$ and $\Delta z$ varies from $0.175 \mathrm{~km}$ at the top to $0.25 \mathrm{~km}$ at the bottom. The $\Delta z$ in each subdomain varies 
linearly with depth as shown in Fig. 6(b). The thickness of the first and second layer is $1.8 \mathrm{~km}$ and $7.5 \mathrm{~km}$, respectively. The calculation of spatial derivatives with respect to $z$ is performed by using the mapping scheme (Fornberg, 1988; Jastram and Tessmer, 1994; Furumura et al., 1998b) since the $\Delta z$ is varying in the $z$ direction.

The seismic source is an explosion point source located at a depth of $15.0 \mathrm{~km}$. The spatial distribution of the body force components and the source time function are approximated by the same type pseudo-delta function as used in the previous examples. The width of the pseudo-delta type source time function is $0.3 \mathrm{~s}$. The time interval $\Delta t$ determined from the minimum value in the three subdomains is $0.004 \mathrm{~s}$. The free-surface and absorbing boundary conditions are the same as in the previous examples.

Figure 7 shows the synthetic seismograms along a vertical profile with horizontal distance of $4.8 \mathrm{~km}$ from the source. Various seismic phases radiated directly from the source, reflected and converted at the free surface and at the interfaces can be identified.

This calculation was performed in single precision on a SUN Ultra 1 workstation with $143 \mathrm{MHz}$ clock speed with memory of 26.37 Mbytes and CPU time of $81.62 \mathrm{~min}$.

We performed the calculation for the same model defined on continuous uniform grid. The grid spacing is $0.05 \mathrm{~km}$ and the number of grid points is 512 for the whole model in the $x$ direction. The same grid spacing and mapping function as used for the discontinuous grid are applied for the grid spacing in the $z$ direction. The time interval used for this calculation is $0.003 \mathrm{~s}$, the memory is 30.12 Mbytes and the CPU time is $188.50 \mathrm{~min}$. In Fig. 8, we show the synthetic seismograms at four locations at the surface, $1.0 \mathrm{~km}, 2.0 \mathrm{~km}$ and $3.0 \mathrm{~km}$ depth along the vertical profile shown in Fig. 7 for both the discontinuous and the uniform grids. The two results agree well in both amplitudes and arrival times for all seismic phases. Compared to the uniform grid, the discontinuous grid takes about $88 \%$ of the computer memory and $43 \%$ of the CPU time.

\section{Conclusions}

When the PSM is used in wave propagation simulation in realistic heterogeneous medium defined on a conventional uniform grid, the grid spacing is determined from the lowest seismic wave velocity in the model. Such a discretized model causes oversampling in space in the region with higher seismic wave velocity, and consequently large computer memory and long CPU time in the case of a large scale model. In this paper, we have proposed the multidomain scheme using a discontinuous grid, in which the grid spacing varies in the model with the changing seismic wave velocity. The scheme overcomes the spatial oversampling in the conventional uniform grid. It keeps the high accuracy while increases efficiency of the PSM by reducing the computer memory and the computation time required for the calculation compared to the conventional uniform grid. This makes it more efficient to apply the PSM to large scale or 3-D model.

We considered the multidomain scheme using discontinuous grid for models defined in 2-D Cartesian coordinates in this paper. Application of this scheme to 3-D model is very straightforward. The present scheme is also applicable to models defined in 2-D cylindrical coordinates (Wang et al., 2001).

Acknowledgments. We are very grateful to Drs. P. Moczo and A. Pitarka for the critical reviews that significantly improved the manuscript. Careful reading and checking of the manuscript by Dr. R. Martin is appreciated. This study was partially supported by the comprehensive joint research project "Master model of strong motion prediction for mitigating earthquake disaster" funded by the coordination fund for promoting science and technology, Ministry of Education, Culture, Sports, Science and Technology of Japan.

\section{References}

Aoi, S. and H. Fujiwara, 3D finite-difference method using discontinuous grids, Bull. Seis. Soc. Am., 89, 918-930, 1999.

Bouchon, M. and K. Aki, Discrete wave-number representation of seismicsource wave fields, Bull. Seis. Soc. Am., 67, 259-277, 1977.

Cerjan, C., D. Kosloff, R. Kosloff, and M. Reshef, A nonreflecting boundary condition for discrete acoustic and elastic wave equations, Geophysics, 50, 705-708, 1985.

Daudt, C., L. Braile, R. Nowack, and C. Chiang, A comparison of finitedifference and Fourier method calculations of synthetic seismogram, Bull. Seis. Soc. Am., 79, 1210-1230, 1989.

Falk, J., E. Tessmer, and D. Gajewski, Tube wave modeling by the finitedifference method with varying grid spacing, Pageoph, 148, 77-93, 1996.

Fornberg, B., The pseudospectral method: Comparisons with finite differences for the elastic wave equation, Geophysics, 52, 483-501, 1987.

Fornberg, B., The pseudospectral method: accurate representation of interfaces in elastic wave calculations, Geophysics, 53, 625-637, 1988.

Furumura, T. and K. Koketsu, Specific distribution of ground motion during the 1995 Kobe earthquake and its generation mechanism, Geophys. Res. Lett., 25, 785-788, 1998.

Furumura, T. and H. Takenaka, A stable method for numerical differentiation of data with discontinuities at end-points by means of Fourier transformsymmetric differentiation, Butsuri-Tansa (J. SEGJ), 45, 303-309, 1992 (in Japanese with English abstract).

Furumura, T., H. Takenaka, and K. Koketsu, Numerical 3-D modeling of seismic waves by the pseudospectral method, Butsuri-Tansa (J. SEGJ), 49, 536-548, 1996 (in Japanese with English abstract).

Furumura, T., B. L. N. Kennett, and H. Takenaka, Parallel 3-D pseudospectral simulation of seismic wave propagation, Geophysics, 63, 279-288, 1998a.

Furumura, T., B. L. N. Kennett, and M. Furumura, Seismic wavefield calculation for laterally heterogeneous whole earth models using the pseudospectral method, Geophys. J. Int., 135, 645-860, 1998 b.

Graves, R. W., Simulating realistic earthquake ground motions in regions of deep sedimentary basins, Proc. of the 11th World Conference on Earthquake Engineering, Acapulco, Mexico, 1996.

Herrmann, R. B., SH-wave generation by dislocation source-a numerical study, Bull. Seis. Soc. Am., 69, 1-15, 1979.

Hung, S. H. and D. W. Forsyth, Modelling anisotropic wave propagation in oceanic inhomogeneous structures using parallel multidomain pseudospectral method, Geophys. J. Int., 133, 726-740, 1998.

Jastram, C. and A. Behle, Acoustic modeling on a grid of vertically varying spacing, Geophys. Prosp., 40, 157-170, 1992.

Jastram, C. and E. Tessmer, Elastic modelling on a grid with vertically varying spacing, Geophys. Prosp., 42, 357-370, 1994.

Kosloff, D. and E. Baysal, Forward modeling by a Fourier method, Geophysics, 47, 1402-1412, 1982.

Kosloff, D., M. Reshef, and D. Loewenthal, Elastic wave calculation by the Fourier method, Bull. Seis. Soc. Am., 74, 875-891, 1984.

Moczo, P., Finite-difference technique for SH-waves in 2-D media using irregular grids-application to the seismic response problem, Geophys. J. Int., 99, 321-329, 1989.

Moczo, P. and P.-Y. Bard, Wave diffraction, amplification and differential motion near strong lateral discontinuities, Bull. Seis. Soc. Am., 83, 85106, 1993.

Moczo, P., P. Labák, J. Kristek, and F. Hron, Amplification and differential motion due to an antiplane $2 \mathrm{D}$ resonance in the sediment valleys embedded in a layer over the half-space, Bull. Seis. Soc. Am., 86, 1434-1446, 1996.

Moczo, P., E. Bystrický, J. Kristek, J. M. Carcione, and M. Bouchon, Hybrid modeling of P-SV seismic motion at inhomogeneous viscoelastic topographic structures, Bull. Seis. Soc. Am., 87, 1305-1323, 1997. 
Olsen, K. B. and R. J. Archuleta, Three-dimensional simulation of earthquakes on the Los Angeles fault system, Bull. Seis. Soc. Am., 86, 575-596, 1996

Olsen, K. B., R. J. Archuleta, and J. R. Matarese, Magnitude 7.75 earthquake on the San Andreas fault: three-dimensional ground motion in Los Angeles, Science, 270, 1628-1632, 1995.

Pitarka, A., 3D elastic finite-difference modeling of seismic motion using staggered grids with nonuniform spacing, Bull. Seis. Soc. Am., 89, 54-68, 1999.

Sato, T., R. W. Graves, P. G. Somerville, and S. Kataoka, Estimates of regional and local strong motions during the great 1923 Kanto, Japan, earthquake $\left(M_{S} 8.2\right)$, part 1: forward simulation of seismograms using variable slip rupture models and estimation of near fault long period ground motions, Bull. Seis. Soc. Am., 88, 206-227, 1998.

Wang, Y. B., H. Takenaka, and T. Furumura, Modelling seismic wave propagation in a 2-D cylindrical whole earth model using the pseudospectral method, Geophys. J. Int., 2001 (in press).

Y. Wang (e-mail: wangy@ipgp.jussieu.fr) and H. Takenaka (e-mail: takenaka@geo.kyushu-u.ac.jp) 